PROCEEDINGS OF THE

AMERICAN MATHEMATICAL SOCIETY

Volume 133, Number 1, Pages 193-202

S 0002-9939(04)07545-8

Article electronically published on June 23, 2004

\title{
NORMS ON EARTHQUAKE MEASURES AND ZYGMUND FUNCTIONS
}

\author{
JUN HU
}

(Communicated by Juha M. Heinonen)

\begin{abstract}
The infinitesimal earthquake theorem gives a one-to-one correspondence between Thurston bounded earthquake measures and normalized Zygmund bounded functions. In this paper, we provide an intrinsic proof of a theorem given in an earlier paper by the author; that is, we show that the cross-ratio norm of a Zygmund bounded function is equivalent to the Thurston norm of the earthquake measure in the correspondence.
\end{abstract}

\section{INTRODUCTION}

Consider the open unit disk $\mathbb{D}$ centered at the origin of the complex plane $\mathbb{C}$ as the hyperbolic plane. A geodesic lamination $\mathcal{L}$ in $\mathbb{D}$ is a collection of geodesics that foliate a closed subset $L$ of $\mathbb{D}$. Here $L$ is called the locus of $\mathcal{L}$, the geodesics are called the leaves of $\mathcal{L}$, the connected components of $\mathbb{D} \backslash L$ are called the gaps, and the gaps and the leaves of $\mathcal{L}$ are called the strata of $\mathcal{L}$. Let $\mathbb{S}^{1}$ denote the boundary circle of $\mathbb{D}$, and let $\mathbb{X}$ be the space $\mathbb{S}^{1} \times \mathbb{S}^{1} \backslash\{$ the diagonal $\}$ factorized by the equivalence relation $(a, b) \sim(b, a)$. A Borel measure $\sigma$ defined on $\mathbb{X}$ is called an earthquake measure if there is a lamination $\mathcal{L}$ such that $\sigma$ is supported on the pairs of the endpoints of the leaves in $\mathcal{L}$.

Let $\mathcal{L}$ be a lamination in $\mathbb{D}$ and $\beta$ a closed geodesic segment of hyperbolic length $\leq 1$. If $\beta$ is transversal to a leaf in $\mathcal{L}$, then $\mathcal{L}$ intersects $\beta$ in a parallel fashion in the sense that there are two geodesic lines $l_{1}$ and $l_{2}$ among the lines of $\mathcal{L}$ intersecting $\beta$ such that any other line in $\mathcal{L}$ intersecting $\beta$ separates $l_{1}$ from $l_{2}$. Suppose the strip $S$ bounded by $l_{1}$ and $l_{2}$ in the unit disk is of the form $[a, b] \times[c, d]$, where $a, d$ and $b, c$ are the endpoints of $l_{1}$ and $l_{2}$, respectively, and $a, b, c, d$ are arranged on $\mathbb{S}^{1}$ in counter-clockwise order. We denote by

$$
\sigma(\beta)=\sigma([a, b] \times[c, d]) .
$$

In [5], Thurston defines the norm of $\sigma$ to be

$$
\|\sigma\|_{T h}=\sup _{l(\beta) \leq 1} \sigma(\beta)=\sup _{l(\beta)=1} \sigma(\beta),
$$

Received by the editors March 14, 2003 and, in revised form, September 19, 2003.

2000 Mathematics Subject Classification. Primary 37E10; Secondary 37F30.

Key words and phrases. Earthquake measures, Zygmund functions.

This work was supported in part by an NSF postdoctoral research fellowship (DMS 9804393), an Incentive Scholar Fellowship of The City University of New York (2000-01) and PSC-CUNY research grants. 
where $\beta$ is a closed geodesic segment transversal to $\mathcal{L}$ and $l(\beta)$ denotes the hyperbolic length of $\beta$. An earthquake measure is called Thurston bounded if it has finite Thurston norm. Let $\mathcal{M}$ be the collection of all Thurston bounded earthquake measures defined on $\mathbb{X} . \forall \sigma \in \mathcal{M}$, define

$$
V_{\sigma}(x)=E(\sigma)(x)=\iint E_{a b}(x) d \sigma(a, b),
$$

where $E_{a b}(x)=\frac{(x-a)(x-b)}{a-b}$ if $x \in[a, b]$ and $E_{a b}(x)=0$ otherwise. Here we also have the agreement that for each geodesic line $\overline{a b}$ in $\mathcal{L},[a, b]$ denotes the short arc on $\mathbb{S}^{1}$ in counter-clockwise order. $V_{\sigma}$ maps each point $x$ on $\mathbb{S}^{1}$ to a vector tangent to $\mathbb{S}^{1}$ at $x$, and then it defines a tangent vector field on $\mathbb{S}^{1}$.

Simply consider the tangent vectors of $\mathbb{S}^{1}$ as complex numbers. A continuous tangent vector field $V$ on $\mathbb{S}^{1}$ is said to be Zygmund bounded if

$$
\left|V\left(e^{2 \pi i(\theta+t)}\right)+V\left(e^{2 \pi i(\theta-t)}\right)-2 V\left(e^{2 \pi i \theta}\right)\right| \leq M|t|
$$

for a constant $M>0$ and for all $0 \leq \theta<1$ and $0<t<\frac{1}{2}$. It is proved in [1] that for any $\sigma \in \mathcal{M}, V_{\sigma}$ is Zygmund bounded; conversely, for any Zygmund bounded tangent vector field $V$ on $\mathbb{S}^{1}$, there exists a Thurston bounded earthquake measure $\sigma$ such that

$$
V(x)=\pi \iint E_{a b}(x) d \sigma(a, b) \text { modulo a quadratic polynomial; }
$$

furthermore, if two $V$ 's differ by a quadratic polynomial, then the corresponding $\sigma$ 's are the same. This is the so-called infinitesimal earthquake theorem (see Theorem 5.1 in 1]), which gives a one-to-one correspondence between $\mathcal{M}$ and the space of Zygmund bounded tangent vector fields on $\mathbb{S}^{1}$.

Motivated by the work of [3, we introduce a norm to measure the Zygmund boundedness of $V$ and show it is equivalent to the Thurston norm of $\sigma$. Given a quadruple $Q=\{a, b, c, d\}$ consisting of four points $a, b, c, d$ on the unit circle $\mathbb{S}^{1}$ arranged in counter-clockwise order, we define

$$
\operatorname{cr}(Q)=\frac{(b-a)(d-c)}{(c-b)(d-a)}
$$

and

$$
V[Q]=\frac{V(b)-V(a)}{b-a}-\frac{V(c)-V(b)}{c-b}+\frac{V(d)-V(c)}{d-c}-\frac{V(a)-V(d)}{a-d} .
$$

Then the cross-ratio norm $\|V\|_{c r}$ of $V$ is defined to be

$$
\|V\|_{c r}=\sup _{c r(Q)=1}|V[Q]| .
$$

In fact, $\|V\|_{c r}$ is finite if and only if $V$ is Zygmund bounded.

It is proved in [2] that $V_{\sigma}$ is the initial derivative to the parameter $t$ of the earthquake curve determined by $t \sigma, t \geq 0$. By means of an implicit method, it is deduced in [4] that the cross-ratio norm of $V_{\sigma}$ is equivalent to the Thurston norm of $\sigma$. On the other hand, it is a general principle that strategies in the study of earthquake theory (see [3] and [2]) transfer to parallel strategies in the infinitesimal theory. In this paper, we construct an intrinsic and direct proof for the following theorem. 
Main Theorem. There exists a universal constant $C>0$ such that $\forall \sigma \in \mathcal{M}$,

$$
\frac{1}{C}\|\sigma\|_{T h} \leq\left\|V_{\sigma}\right\|_{c r} \leq C\|\sigma\|_{T h}
$$

\section{ProOF}

We divide the proof into two parts. We first prove that there exists $C>0$ such that $\|\sigma\|_{T h} \leq C\left\|V_{\sigma}\right\|_{c r}$; we then show that there exists $C>0$ such that $\left\|V_{\sigma}\right\|_{c r} \leq C\|\sigma\|_{T h}$. Before we start the proofs, let us summarize some techniques into lemmas.

Let $\sigma$ denote a Thurston bounded earthquake measure, and let $V=V_{\sigma}$. Let $B$ denote an orientation-preserving Möbius transformation from the upper half-plane $\mathbb{H}$ or the unit open disk $\mathbb{D}$ onto $\mathbb{D}$, and let $\tilde{\sigma}=B_{*}^{-1}(\sigma)$ be the pullback of $\sigma$ under $B$ (i.e., the pushforward of $\sigma$ under $B^{-1}$ ). Also, define

$$
\tilde{V}(x)=V_{\tilde{\sigma}}(x)=E(\tilde{\sigma})(x)=\iint E_{a b}(x) d \tilde{\sigma}(a, b) .
$$

Lemma 1.

$$
\tilde{V}(x)=\frac{V(B(x))}{B^{\prime}(x)} .
$$

Proof. Define $x^{\prime}=B(x), a^{\prime}=B(a)$ and $b^{\prime}=B(b)$. By using the identity

$$
[B(a)-B(b)]^{2}=(a-b)^{2} B^{\prime}(a) B^{\prime}(b)
$$

and the assumption that $B$ is orientation-preserving, we have

$$
\frac{[B(x)-B(a)][B(x)-B(b)]}{[B(a)-B(b)]}=\frac{B\left({ }^{\prime} x\right)(x-a)(x-b)}{(a-b)} .
$$

Then

$$
\begin{aligned}
V(B(x)) & =V\left(x^{\prime}\right)=\iint E_{a^{\prime} b^{\prime}}\left(x^{\prime}\right) d \sigma\left(a^{\prime}, b^{\prime}\right)=\iint \frac{\left(x^{\prime}-a^{\prime}\right)\left(x^{\prime}-b^{\prime}\right)}{a^{\prime}-b^{\prime}} d \sigma\left(a^{\prime}, b^{\prime}\right) \\
& =\iint \frac{[B(x)-B(a)][B(x)-B(b)]}{B(a)-B(b)} d \sigma(B(a), B(b)) \\
& =\iint \frac{B^{\prime}(x)(x-a)(x-b)}{(a-b)} d \tilde{\sigma}(a, b)=B^{\prime}(x) \tilde{V}(x) .
\end{aligned}
$$

Therefore,

$$
\tilde{V}(x)=\frac{V(B(x))}{B^{\prime}(x)} .
$$

Lemma 2. For any quadruple $Q$ of four points $a, b, c, d$ on the real line or the unit circle in counter-clockwise order,

$$
\tilde{V}[Q]=V[B(Q)] \quad\left(\text { or } V[Q]=\tilde{V}\left[B^{-1}(Q)\right]\right) .
$$

Proof. Let $h_{t}=I d+t V$, where $t$ is a real parameter near zero. Clearly, for each $x$, $h_{t}(x)$ is differentiable on $t$ with $V=\left.\frac{d}{d t} h_{t}\right|_{t=0}$. Define

$$
\operatorname{cr}\left(h_{t}(Q)\right)=\frac{\left[h_{t}(b)-h_{t}(a)\right]\left[h_{t}(d)-h_{t}(c)\right]}{\left[h_{t}(c)-h_{t}(b)\right]\left[h_{t}(d)-h_{t}(a)\right]} .
$$

Observe first that

$$
V[Q]=\left.\frac{d}{d t} \ln \operatorname{cr}\left(h_{t}(Q)\right)\right|_{t=0}
$$


Let $\tilde{h}_{t}=B^{-1} \circ h_{t} \circ B$. Then $\tilde{h}_{t}$ is also differentiable on $t$, and

$$
\left.\frac{d}{d t} \tilde{h}_{t}(x)\right|_{t=0}=\frac{V(B(x))}{B^{\prime}(x)}=\tilde{V}(x) .
$$

Therefore,

$$
\tilde{V}[Q]=\left.\frac{d}{d t} \ln \operatorname{cr}\left(\tilde{h}_{t}(Q)\right)\right|_{t=0}
$$

On the other hand,

$$
\operatorname{cr}\left(\tilde{h}_{t}(Q)\right)=\operatorname{cr}\left(B^{-1} \circ h_{t} \circ B(Q)\right)=\operatorname{cr}\left(h_{t} \circ B(Q)\right)=\operatorname{cr}\left(h_{t}(B(Q))\right),
$$

and then

$$
\left.\frac{d}{d t} \ln c r\left(\tilde{h}_{t}(Q)\right)\right|_{t=0}=\left.\frac{d}{d t} \ln \operatorname{cr}\left(h_{t}(B(Q))\right)\right|_{t=0}=V[B(Q)]
$$

Hence

$$
\tilde{V}[Q]=V[B(Q)]
$$

Corollary 1. $\|\tilde{V}\|_{c r}=\|V\|_{c r}$.

Lemma 3. Assume $\lambda>0,-\infty \leq a<b<c<d$, and $c \leq s \leq d \leq t$. Let $V(x)=\lambda E_{s, t}(x)$ and $Q=\{a, b, c, d\}$. Consider $V[Q]$ as a function of $s$ and $t$. Then $V[Q] \geq 0$, and $V[Q]$ is an increasing function on $t$ for each fixed $s$ and $a$ decreasing function on $s$ for each fixed $t$.

Proof. It is easy to check that $\frac{\partial}{\partial t} E_{s, t}(x)>0$ and $\frac{\partial}{\partial s} E_{s, t}(x)<0$ for each $s<x<t$. Clearly,

$$
\begin{aligned}
V[Q] & =\frac{V(b)-V(a)}{b-a}-\frac{V(c)-V(b)}{c-b}+\frac{V(d)-V(c)}{d-c}-\frac{V(a)-V(d)}{a-d} \\
& =\frac{V(d)}{d-c}-\frac{V(d)}{d-a}=\left(\frac{1}{d-c}-\frac{1}{d-a}\right) \lambda E_{s, t}(d) .
\end{aligned}
$$

Since $\frac{1}{d-c}-\frac{1}{d-a}>0$,

$$
\frac{\partial}{\partial t} V[Q]>0 \text { and } \frac{\partial}{\partial s} V[Q]<0 .
$$

Therefore $V[Q]$ is an increasing function on $t$ for each fixed $s$ and is a decreasing function on $s$ for each fixed $t$.

Lemma 4. Assume $\lambda>0,-\infty \leq a<b<c<d \leq \infty$, and $b \leq s \leq c$ and $t \geq d$. Let $V(x)=\lambda E_{s, t}(x)$ and $Q=\{a, b, c, d\}$. Consider $V[Q]$ as a function of $s$ and $t$. Then $V[Q] \geq 0$, and $V[Q]$ is increasing on s for each fixed $t$ and also increasing on $t$ for each fixed $s$.

Proof. It is straightforward to check

$$
\begin{aligned}
V[Q] & =\lambda\left[-\frac{d-b}{(c-b)(d-c)} E_{s, t}(c)+\frac{c-a}{(d-c)(d-a)} E_{s, t}(d)\right] \\
& =\lambda\left[-\frac{d-b}{(c-b)(d-c)} \frac{(c-s)(c-t)}{s-t}+\frac{c-a}{(d-c)(d-a)} E_{s, t}(d) \frac{(d-s)(d-t)}{s-t}\right] .
\end{aligned}
$$


Then

$$
\begin{aligned}
\frac{\partial}{\partial s} V[Q] & =\lambda\left[\frac{(d-b)}{(c-b)(d-c)} \frac{(t-c)^{2}}{(s-t)^{2}}-\frac{(c-a)}{(d-c)(d-a)} \frac{(t-d)^{2}}{(s-t)^{2}}\right] \\
& =\frac{\lambda(t-d)^{2}}{(s-t)^{2}(d-c)}\left[\frac{d-b}{c-b}\left(\frac{t-c}{t-d}\right)^{2}-\frac{c-a}{d-a}\right] .
\end{aligned}
$$

Clearly, $\frac{d-b}{c-b}\left(\frac{t-c}{t-d}\right)^{2}>1$ and $\frac{c-a}{d-a}<1$. Hence $\frac{\partial}{\partial s} V[Q]>0$ when $t>d$ and $\frac{\partial}{\partial s} V[Q] \geq$ 0 when $t=d$. Therefore $V[Q]$ is an increasing function on $s$ for each fixed $t$.

Similarly,

$$
\begin{aligned}
\frac{\partial}{\partial t} V[Q] & =\lambda\left[-\frac{(d-b)}{(c-b)(d-c)} \frac{(c-s))^{2}}{(s-t)^{2}}+\frac{(c-a)}{(d-c)(d-a)} \frac{(d-s)^{2}}{(s-t)^{2}}\right] \\
& =\frac{\lambda(c-s)^{2}}{(s-t)^{2}(d-c)}\left[-\frac{d-b}{c-b}+\frac{c-a}{d-a}\left(\frac{d-s}{c-s}\right)^{2}\right] \\
& =\frac{\lambda(c-s)^{2}}{(s-t)^{2}(d-c)} \frac{d-b}{c-b}\left[-1+\left(\frac{d-s}{c-s} / \frac{d-b}{c-b}\right)\left(\frac{d-s}{c-s} / \frac{d-a}{c-a}\right)\right] .
\end{aligned}
$$

Since $a<b \leq s \leq c<d$,

Then

$$
\frac{d-s}{c-s} \geq \frac{d-b}{c-b}>1 \text { and } \frac{d-s}{c-s}>\frac{d-a}{c-a}>1 .
$$

$$
-1+\left(\frac{d-s}{c-s} / \frac{d-b}{c-b}\right)\left(\frac{d-s}{c-s} / \frac{d-a}{c-a}\right)>0 .
$$

Hence $\frac{\partial}{\partial t} V[Q]>0$ when $b \leq s<c$ and $\frac{\partial}{\partial t} V[Q]=0$ when $s=c$. Therefore $V[Q]$ is also an increasing function on $t$ for each fixed $s$.

Theorem 1. There exists a universal constant $C>0$ such that

$$
\|\sigma\|_{T h} \leq C\left\|V_{\sigma}\right\|_{c r}
$$

In fact, we can take $C=\frac{(1+e)^{2}}{-3 e^{2}+6 e+1}$.

Proof. Let $D$ denote a closed disk in $\mathbb{D}$ of hyperbolic diameter $1, l_{1}$ and $l_{2}$ denote the lines in the lamination $\mathcal{L}$ of $\sigma$ that bound all the lines in $\mathcal{L}$ intersecting $D$. Let $\beta$ denote the geodesic perpendicular to both $l_{1}$ and $l_{2}$ (in the case that $l_{1}$ and $l_{2}$ share at least one endpoint, we let $\beta$ be a geodesic perpendicular to $l_{1}$ such that the hyperbolic length of the segment on $\beta$ between $l_{1}$ and $l_{2}$ is less than or equal to $\frac{1}{2}$ ). Label the endpoints of $\beta$ by $x$ and $y$ so that the arc $[x, y]$ from $x$ to $y$ going in the counter-clockwise direction is not longer than the arc $[y, x]$ from $y$ to $x$. Let $B: \mathbb{D} \rightarrow \mathbb{H}$ be the Möbius transformation that maps $x$ to $0, y$ to $\infty$, the arc $[x, y]$ to the positive half real line, and the geodesics $l_{1}$ and $l_{2}$ to the geodesics connecting -1 to 1 and $-s$ to $s$ with $s>1$ (in the case that $l_{1}$ and $l_{2}$ share at least one endpoint, then $B\left(l_{1}\right)$ or $B\left(l_{2}\right)$ connects $-s$ to 1 or -1 to $s$ with $\left.s \geq 1\right)$. Since the hyperbolic distance between $l_{1}$ and $l_{2}$ is less than or equal to $1, s \leq e$ (in the case that $l_{1}$ and $l_{2}$ share one endpoint, the requirment on $\beta$ also implies that $s \leq e$ ). Let $\tilde{\sigma}$ denote the pushforward of $\sigma$ under $B$ and $\tilde{V}=E(\tilde{\sigma})$. It is clear that $\|\tilde{\sigma}\|_{T h}=\|\sigma\|_{T h}$, and Lemma 2 implies $\|\tilde{V}\|_{c r}=\|V\|_{T h}$.

Assume $a=1, b=\infty, c=-s$ and $d=\frac{1-s}{2}$. Denote by $Q=\{a, b, c, d\}$ and $Q^{\prime}=B^{-1}(Q)=\left\{a^{\prime}, b^{\prime}, c^{\prime}, d^{\prime}\right\}$. Clearly, $\operatorname{cr}(Q)=\operatorname{cr}\left(Q^{\prime}\right)=1$, and Lemma 2 implies $\tilde{V}[Q]=V\left[Q^{\prime}\right]$. Denote by $u^{\prime}$ and $v^{\prime}$ the other endpoints of $l_{1}$ and $l_{2}$ such that $a^{\prime}, u^{\prime}, b^{\prime}, c^{\prime}, v^{\prime}, d^{\prime}$ are arranged on $\mathbb{S}^{1}$ in counter-clockwise order. We divide the lines 
in the lamination $\mathcal{L}$ that affect the value of $V\left[Q^{\prime}\right]$ into three groups. Let $\mathcal{L}_{m}$ denote the collection of the lines in $\mathcal{L}$ intersecting $D, \mathcal{L}_{b}$ denote the collection of the lines in $\mathcal{L} \backslash \mathcal{L}_{m}$ connecting points in $\left[u^{\prime}, b^{\prime}\right)$ to points in $\left(b^{\prime}, c^{\prime}\right]$, and $\mathcal{L}_{d}$ the collection of the lines in $\mathcal{L} \backslash \mathcal{L}_{m}$ connecting points in $\left[v^{\prime}, d^{\prime}\right)$ to points in $\left(d^{\prime}, a^{\prime}\right]$. Denote by $\sigma_{i}=\left.\sigma\right|_{\mathcal{L}_{i}}$ and $V_{i}=E\left(\sigma_{i}\right)$ for $i=m, b, d$. By the linearity of the operator $E$, we have

$$
V\left[Q^{\prime}\right]=V_{m}\left[Q^{\prime}\right]+V_{b}\left[Q^{\prime}\right]+V_{d}\left[Q^{\prime}\right]
$$

By Lemma 3

$$
V_{d}\left[Q^{\prime}\right]=V_{d}\left[\left\{a^{\prime}, b^{\prime}, c^{\prime}, d^{\prime}\right\}\right] \geq 0 \text { and } V_{b}\left[\left\{c^{\prime}, d^{\prime}, a^{\prime}, b^{\prime}\right\}\right] \geq 0 .
$$

Then

$$
V_{b}\left[Q^{\prime}\right]=V_{b}\left[\left\{a^{\prime}, b^{\prime}, c^{\prime}, d^{\prime}\right\}\right]=-V_{b}\left[\left\{b^{\prime}, c^{\prime}, d^{\prime}, a^{\prime}\right\}\right]=V_{b}\left[\left\{c^{\prime}, d^{\prime}, a^{\prime}, b^{\prime}\right\}\right] \geq 0 .
$$

Therefore,

$$
V\left[Q^{\prime}\right] \geq V_{m}\left[Q^{\prime}\right]
$$

To complete the proof, we need to work out an explicit lower bound for $V_{m}\left[Q^{\prime}\right]$. Denote by $\tilde{\sigma}_{m}$ the pushforward of $\sigma_{m}$ under $B$ and $\tilde{V}_{m}=E\left(\tilde{\sigma}_{m}\right)$. By Lemma2,

$$
V_{m}\left[Q^{\prime}\right]=\tilde{V}_{m}\left[B\left(Q^{\prime}\right)\right]=\tilde{V}_{m}[Q] .
$$

By Lemma 4, if we move the weights of the geodesic lines in the lamination $\tilde{\mathcal{L}}_{m}$ of $\tilde{\sigma}_{m}$ to the geodesic line connecting -1 to $s$, then the value of $\tilde{V}_{m}[\{b, c, d, a\}]$ is possibly increased, and hence the value of $\tilde{V}_{m}[Q]=-\tilde{V}_{m}[\{b, c, d, a\}]$ is possibly decreased. Therefore

$$
\tilde{V}_{m}[Q] \geq\left(\lambda E_{-1, s}\right)[Q]
$$

where $\lambda=\sigma\left(\mathcal{L}_{m}\right)$. It is easy to check that

$$
\left(\lambda E_{-1, s}\right)[Q]=\lambda \frac{2 E_{-1, s}(d)-E_{-1, s}(a)}{a-d}
$$

and

$$
\frac{2 E_{-1, s}(d)-E_{-1, s}(a)}{a-d}=\frac{-3 s^{2}+6 s+1}{(1+s)^{2}} \geq \frac{-3 e^{2}+6 e+1}{(1+e)^{2}}>0
$$

for $1 \leq s \leq e$. Letting $C=\frac{(1+e)^{2}}{-3 e^{2}+6 e+1}$, we have

$$
\|V\|_{c r} \geq V\left[Q^{\prime}\right] \geq V_{m}\left[Q^{\prime}\right]=\tilde{V}_{m}[Q] \geq\left(\lambda E_{-1, s}\right)[Q] \geq \frac{\lambda}{C} .
$$

Hence

which implies

$$
\lambda \leq C\|V\|_{c r}
$$

$$
\|\sigma\|_{T h} \leq C\|V\|_{c r}
$$

Theorem 2. There exists a universal constant $C>0$, independent of $\sigma$, such that

$$
\left\|V_{\sigma}\right\|_{c r} \leq C\|\sigma\|_{T h} .
$$

In fact, we can take $C=C_{0}+2 C_{1} C_{2}$, where $C_{0}$ is the smallest positive integer greater than or equal to $\ln (3+2 \sqrt{2}), C_{1}=\frac{e}{e-1}$, and $C_{2}$ is the smallest positive integer greater than or equal to $\ln \left(e+\sqrt{e^{2}-1}\right)$. 
Remark. The inequality (10) was obtained in [1] for a different cross-ratio norm on $V$ through a complex method which considered the holomorphic differential arising from the third derivative of $V+i H(V)$, where $H(V)$ denotes the Hilbert transformation of $V$. The proof of the inequality (10) in this paper is purely real. In addition, our Theorem 1 and that inequality in [1] imply that the two cross-ratio norms on $V$ are actually equivalent (see [4]).

Lemma 5. Let $Q$ be a quadruple consisting of four points $a, b, c, d$ on $\mathbb{R}^{1}$ or $\mathbb{S}^{1}$ arranged in counter-clockwise order. Take $\frac{|d z|}{y}$ as the hyperbolic metric on the upper half-plane $\mathbb{H}$. Then $\operatorname{cr}(Q)=1$ if and only if the geodesic $\overline{\text { ac }}$ from a to $c$ is perpendicular to the geodesic $\overline{b d}$ from $b$ to $d$, and if and only if the hyperbolic distance from $\overline{a b}$ to $\overline{c d}$ (or $\overline{b c}$ to $\overline{d a}$ ) is equal to $\ln (3+2 \sqrt{2})$.

Proof. It is straightforward (see [3] for details).

Lemma 6. Consider the upper half-plane $\mathbb{H}$. Let $l_{n}$ denote the geodesic connecting $-e^{-n}$ to $e^{-n}$ for each $n \in\{0\} \cup \mathbb{N}$ and $\mathcal{L}$ the lamination consisting of $l_{n}$ 's. Suppose that $\sigma$ is an earthquake measure supported on $\mathcal{L}$, and let $\lambda_{n}=\sigma\left(l_{n}\right)$. Let $Q=$ $\{1, \infty,-1,0\}$, and

$$
V(x)=\iint E_{a, b}(x) d \sigma(a, b) .
$$

There exists a constant $C_{1}>0$ such that

$$
0 \leq V[Q] \leq C_{1} \max _{n \geq 0} \lambda_{n} .
$$

Proof. Define $\lambda=\max _{n \geq 0} \lambda_{n}, a_{n}=-e^{-n}$ and $b_{n}=e^{-n}$. Clearly, $V(1)=V(\infty)=$ $V(-1)=0$, and then $V[Q]=2 V(0)$. We need to work out $V(0)$, that is,

$$
0 \leq V(0)=\iint E_{a_{n}, b_{n}}(0) d \sigma\left(a_{n}, b_{n}\right)=\sum_{n \geq 0} \lambda_{n} \frac{a_{n} b_{n}}{b_{n}-a_{n}}=\sum_{n \geq 0} \lambda_{n} \frac{e^{-n}}{2} \leq \frac{1}{2} \frac{e}{e-1} \lambda .
$$

Let $C_{1}=\frac{e}{e-1}$. Then $0 \leq V[Q]=2 V(0) \leq C_{1} \lambda$.

We reduce the proof of Theorem 2 to Propositions 1 and 2 Let $\mathcal{L}$ be the lamination that supports $\sigma$. Given a quadruple $Q$ of four points $a, b, c, d$ on $\mathbb{S}^{1}$ in counter-clockwise order with $\operatorname{cr}(Q)=1$, we first assume that three points $a, b$ and $c$ belong to the same stratum $A$ and estimate $V[Q]$ in this case. By a Möbius change of coordinates and Lemma 2 we may assume that $a=1, b=\infty, c=-1$, and $d=0$. We will show that there is a constant $C$ such that

$$
0 \leq V[Q] \leq C\|\sigma\|_{T h} .
$$

Let $x_{n}$ denote the point $-e^{-n}$ and $y_{n}$ the point $e^{-n}$ on the real axis for each $n \in\{0\} \cup \mathbb{N}$. Let $\mathcal{L}^{\prime}$ denote the collection of the lines in $\mathcal{L}$ that connect points of the interval $[-1,0)$ to points of $(0,1], \mathcal{L}_{0}^{-}$the collection of the lines in $\mathcal{L}^{\prime}$ that connect points of $\left[x_{0}, x_{1}\right)$ to points of $\left(0, y_{0}\right]$, and $\mathcal{L}_{0}^{+}$the collection of the lines in $\mathcal{L}^{\prime}$ that connect points of $\left[x_{0}, 0\right)$ to points of $\left(y_{1}, y_{0}\right]$. Finally, let $\mathcal{L}_{0}=\mathcal{L}_{0}^{-} \cup \mathcal{L}_{0}^{+}$. Then any line in $\mathcal{L}^{\prime} \backslash \mathcal{L}_{0}$ must connect a point in $\left[x_{1}, 0\right)$ to a point in $\left(0, y_{1}\right]$. Inductively, for each $n \in \mathbb{N}$, let $\mathcal{L}_{n}^{-}$denote the collection of the lines in $\mathcal{L}^{\prime} \backslash\left(\mathcal{L}_{0} \cup \mathcal{L}_{1} \cup \cdots \cup \mathcal{L}_{n-1}\right)$ that connect points of $\left[x_{n}, x_{n+1}\right)$ to points of $\left(0, y_{n}\right]$, and $\mathcal{L}_{n}^{+}$the collection of the lines in $\mathcal{L}^{\prime} \backslash\left(\mathcal{L}_{0} \cup \mathcal{L}_{1} \cup \cdots \mathcal{L}_{n-1}\right)$ that connect points of $\left[x_{n}, 0\right)$ to points of $\left(y_{n+1}, y_{n}\right]$, and $\mathcal{L}_{n}=\mathcal{L}_{n}^{-} \cup \mathcal{L}_{n}^{+}$. We have the following three lemmas. 
Lemma 7. For each $n \in\{0\} \cup \mathbb{N}$, any line in $\mathcal{L}_{n}$ must connect a point in $\left[x_{n}, 0\right)$ to a point in $\left(0, y_{n}\right]$.

Proof. It can be easily proved by an induction on $n$.

Lemma 8. There exists a constant $C_{2}>0$, independent of $h$ and $\sigma$, such that $\sigma\left(\mathcal{L}_{n}\right) \leq C_{2}\|\sigma\|_{T h}$ for any $n \in\{0\} \cup \mathbb{N}$.

Proof. For each $n \in\{0\} \cup \mathbb{N}$, let $l_{n}$ denote the geodesic line connecting the point $x_{n}$ to the point $y_{n}$. Also, for any $n \in \mathbb{N}$, let $l_{n}^{-}$denote the geodesic connecting the point $x_{n}$ to 0 , and $l_{n}^{+}$the geodesic connecting 0 to the point $y_{n}$. The hyperbolic distance from $l_{n}$ to $l_{n+1}^{-}\left(\right.$or $\left.l_{n+1}^{+}\right), n \in\{0\} \cup \mathbb{N}$, is equal to a constant, that is equal to $\ln \left(e+\sqrt{e^{2}-1}\right)$. Let $C_{2}$ denote the smallest positive integer that is greater than or equal to $\ln \left(e+\sqrt{e^{2}-1}\right)$. Then

$$
\sigma\left(\mathcal{L}_{n}\right) \leq C_{2}\|\sigma\|_{T h}
$$

for each $n \in\{0\} \cup \mathbb{N}$.

Let $\tilde{V}$ be the same map as defined in Lemma 6 with $\lambda_{n}=\sigma\left(\mathcal{L}_{n}\right)$.

Lemma 9. We have the following inequality:

$$
V[Q] \leq \tilde{V}[Q]
$$

Proof. Define $\sigma_{n}=\left.\sigma\right|_{\mathcal{L}_{n}}$ and $V_{n}=E\left(\sigma_{n}\right)$. Let $\tilde{\sigma}_{n}$ denote the atomic earthquake measure with weight $\lambda_{n}$ supported on the geodesic $l_{n}$ and let $\tilde{V}_{n}=E\left(\tilde{\sigma}_{n}\right)$. By the linearity of the operator $E$,

$$
V[Q]=\sum_{n=0}^{\infty} V_{n}[Q] \text { and } \tilde{V}[Q]=\sum_{n=0}^{\infty} \tilde{V}_{n}[Q] .
$$

By Lemma 3 and Lemma 7 , if we move the weights of the geodesic lines in $\mathcal{L}_{n}$ to the goedesic line $l_{n}$, we only increase $V_{n}[Q]$, that is, $V_{n}[Q] \leq \tilde{V}_{n}[Q]$. Therefore

$$
V[Q] \leq \tilde{V}[Q] .
$$

Lemmas 9, 6 and 8 imply the following proposition.

Proposition 1. If $\operatorname{cr}(Q)=1$ and $a, b, c$ belong to the same stratum of an earthquake measure $(E, \mathcal{L})$, then

$$
0 \leq V_{\sigma}[Q] \leq C_{1} C_{2}\|\sigma\|_{T h} .
$$

Proposition 2. Suppose $\operatorname{cr}(Q)=1$, and assume that there exists at least one geodesic line in the lamination $\mathcal{L}$ of $\sigma$ that separates the vertices a and $b$ from the vertices $c$ and $d$. Then

$$
\left|V_{\sigma}[Q]\right| \leq\left(C_{0}+2 C_{1} C_{2}\right)\|\sigma\|_{T h}
$$

Proof. Given two points $x$ and $y$ on the unit circle, we use $[x, y]$ to denote the arc on $\mathbb{S}^{1}$ from $x$ to $y$ in the counter-clockwise direction. We divide the geodesic lines in $\mathcal{L}$ that affect $V[Q]$ into five groups. Let $\mathcal{L}_{m}$ denote the collection of the geodesic lines in $\mathcal{L}$ that connect points of the $\operatorname{arc}[d, a]$ to points of the arc $[b, c]$. Let $\mathcal{L}_{a}$ denote the collection of the lines in $\mathcal{L}$ that connect points of the arc $(d, a)$ to points of the arc $(a, b), \mathcal{L}_{b}$ the collection of the lines in $\mathcal{L}$ that connect points of the arc $(a, b)$ to points of the $\operatorname{arc}(b, c), \mathcal{L}_{c}$ the collection of the lines in $\mathcal{L}$ that connect 


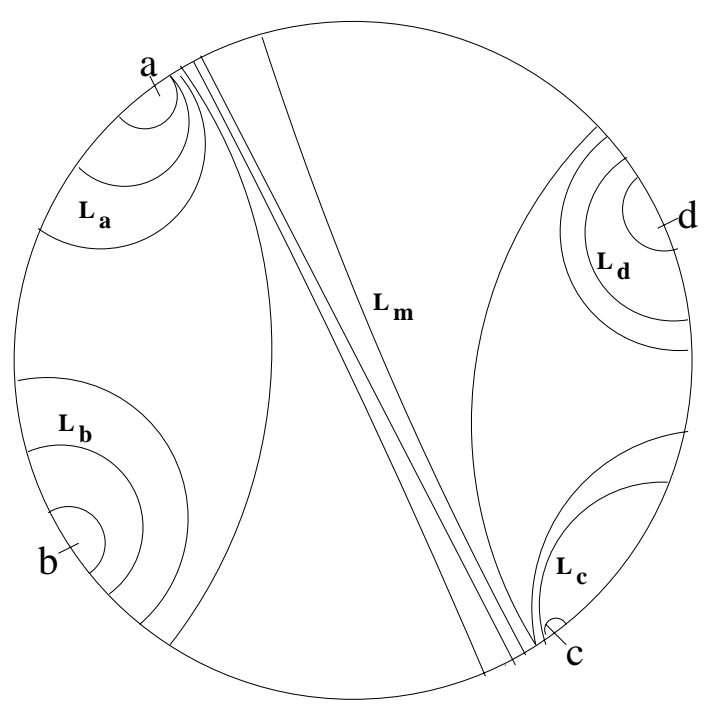

Figure 1. Five subcollections of $\mathcal{L}$ in the proof of Proposition 2

points of the arc $(b, c)$ to points of the arc $(c, d)$, and finally $\mathcal{L}_{d}$ the collection of the lines in $\mathcal{L}$ that connect points of the arc $(c, d)$ to points of the arc $(d, a)$ (see Figure 1 ). Let $\sigma_{i}$ denote the restriction of $\sigma$ on $\mathcal{L}_{i}$ and $V_{i}=E\left(\sigma_{i}\right)$, where $i=m, a, b, c, d$. Clearly $\left\|\sigma_{i}\right\|_{T h} \leq\|\sigma\|_{T h}$ and

$$
V[Q]=V_{m}[Q]+V_{a}[Q]+V_{b}[Q]+V_{c}[Q]+V_{d}[Q] .
$$

For the lamination $\mathcal{L}_{d}$, three points $a, b, c$ are contained in the same stratum. By Proposition 1, we have

$$
0 \leq V_{d}[Q] \leq C_{1} C_{2}\left\|\sigma_{d}\right\|_{T h} \leq C_{1} C_{2}\|\sigma\|_{T h} .
$$

For the lamination $\mathcal{L}_{b}$, three points $c, d, a$ are contained in the same stratum. Again by Proposition 1, we have

$$
0 \leq V_{b}[\{c, d, a, b\}] \leq C_{1} C_{2}\left\|\sigma_{b}\right\|_{T h} \leq C_{1} C_{2}\|\sigma\|_{T h} .
$$

Therefore,

$$
0 \leq V_{b}[Q]=V_{b}[\{c, d, a, b\}] \leq C_{1} C_{2}\|\sigma\|_{T h}
$$

Similarly, we obtain

$$
-C_{1} C_{2}\|\sigma\|_{T h} \leq V_{a}[Q] \leq 0 \text { and }-C_{1} C_{2}\|\sigma\|_{T h} \leq V_{c}[Q] \leq 0 .
$$

It remains to consider $V_{m}[Q]$. Because of Lemma 2, by a Möbius change of coordinates, we may assume $a=-\infty, b=-1, c=0, d=1$. Then the geodesic lines in $\mathcal{L}_{m}$ connect the points of $[-1,0]$ to the points of $[1,+\infty]$. By Lemma 4, if we move all the lines in $\mathcal{L}_{m}$ to the geodesic line from 0 to $\infty$ without changing the weights of the lines in $\mathcal{L}_{m}$ to obtain a new measure $\sigma_{m}^{\prime}$, then $V_{m}^{\prime}[Q]=E\left(\sigma_{m}^{\prime}\right)[Q]$ is possibly bigger, that is, $V_{m}[Q] \leq V_{m}^{\prime}[Q]$. Clearly, $V_{m}^{\prime}(x)=0$ for $x \in[-\infty, 0]$ and $V_{m}^{\prime}(x)=\sigma\left(\mathcal{L}_{m}\right) x$ for $x \in(0,+\infty)$. Then $V_{m}^{\prime}[Q]=V_{m}^{\prime}(1)=\sigma\left(\mathcal{L}_{m}\right)$. If we let $C_{0}$ 
denote the smallest integer $\geq \ln (3+2 \sqrt{2})$, then by Lemma 5 , $\sigma\left(\mathcal{L}_{m}\right) \leq C_{0}\|\sigma\|_{T h}$. Therefore,

$$
V_{m}[Q] \leq V_{m}^{\prime}[Q]=\sigma\left(\mathcal{L}_{m}\right) \leq C_{0}\|\sigma\|_{T h} .
$$

Again by Lemma 4, if we move all the lines in $\mathcal{L}_{m}$ to the geodesic line from -1 to 1 without changing the weights of the lines in $\mathcal{L}_{m}$ to obtain a new measure $\sigma_{m}^{\prime \prime}$, then $V_{m}^{\prime \prime}[Q]=E\left(\sigma_{m}^{\prime \prime}\right)[Q]$ is possibly smaller, that is, $V_{m}[Q] \geq V_{m}^{\prime \prime}[Q]$. Clearly, $V_{m}^{\prime \prime}(x)=0$ for $x \notin(-1,1)$ and $V_{m}^{\prime \prime}(x)=\sigma\left(\mathcal{L}_{m}\right) \frac{(x+1)(1-x)}{2}$ for $x \in(-1,1)$. Then $V_{m}^{\prime \prime}[Q]=-2 V_{m}^{\prime \prime}(0)=-\sigma\left(\mathcal{L}_{m}\right)$. Therefore,

$$
V_{m}[Q] \geq V_{m}^{\prime \prime}[Q]=-\sigma\left(\mathcal{L}_{m}\right) \geq-C_{0}\|\sigma\|_{T h} .
$$

Collecting together these estimates, we obtain

$-\left(C_{0}+2 C_{1} C_{2}\right)\|\sigma\|_{T h} \leq V_{a}[Q]+V_{c}[Q]+V_{m}[Q]+V_{b}[Q]+V_{d}[Q] \leq\left(C_{0}+2 C_{1} C_{2}\right)\|\sigma\|_{T h} ;$

that is,

$$
|V[Q]| \leq\left(C_{0}+2 C_{1} C_{2}\right)\|\sigma\|_{T h}
$$

Propositions 1 and 2 imply Theorem 2 .

Proof. Consider the pattern of the geodesic lines in $\mathcal{L}$ with respect to $Q$, there either exists a line in $\mathcal{L}$ separating two adjacent vertices in $Q$ from the other two or no such line exists. The former case is treated by Proposition 2 and the fact that $V[\{b, c, d, a\}]=-V[Q]$; the latter one is treated by Proposition 1 and the summability of $V[Q]=E(\sigma)[Q]$ over four subcollections of $\mathcal{L}$ that contain three vertices of $Q$ in the same stratum.

Finally, Theorems 1 and 2 imply our Main Theorem.

\section{ACKNOWLEDGEMENT}

The author wishes to thank Prof. Frederick P. Gardiner and the referee for their useful comments to improve the presentation of the paper.

\section{REFERENCES}

[1] F. P. Gardiner. Infinitesimal bending and twisting in one-dimensional dynamics. Trans. Amer. Math. Soc., 347 (3), 915-937, 1995. MR95e:30024

[2] F. P. Gardiner, J. Hu and N. Lakic. Earthquake curves. Contemporary Mathematics, Vol. 311, 141-195, 2002. MR2003i:37033

[3] J. Hu. Earthquake measure and cross-ratio distortion. IMS Preprint \#2001/8, SUNY at Stony Brook (www.math.sunysb.edu/preprints.html, to appear in Contemporary Mathematics).

[4] - On a norm of tangent vectors to earthquake curves. Preprint, Dept. of Math. at Brooklyn College of CUNY, Jan. 2003 (to appear in Advances in Mathematics, Sinica).

[5] W. P. Thurston. Earthquakes in two-dimensional hyperbolic geometry. In Low-dimensional Topology and Kleinian groups, Vol. 112, pp. 91-112. London Math. Soc. Lecture Note Ser., no. 112,1986 . MR:88m:57015

Department of Mathematics, Brooklyn College, Cuny, Brooklyn, New York 11210

E-mail address: jun@sci.brooklyn.cuny.edu 